\title{
Time-invariant entanglement and sudden death of nonlocality for multipartite systems under collective dephasing
}

\author{
MAZHAR Ali \\ Department of Electrical Engineering, Faculty of Engineering, Islamic University Madinah, 107 Madinah, Saudi Arabia
}

PACS 03.67.-a - Quantum information

PACS 03.65.Yz - Decoherence; open systems

PACS 03.65.Ud - Entanglement and quantum nonlocality

\begin{abstract}
We investigate the dynamics of entanglement and nonlocality for multipartite quantum systems under collective dephasing. Using an exact and computable measure for genuine entanglement, we demonstrate the possibility of a non trivial phenomenon of time-invariant entanglement for multipartite quantum systems. We find that for four qubits, there exist quantum states, which are changing continously nevertheless their genuine entanglement remains constant. Based on our numerical results, we conjecture that there is no evidence of time-invariant entanglement for quantum states of three qubits. We point out that quantum states exhibiting time-invariant entanglement must live in both decoherence free subspace and in the subspaces orthogonal to it. The previous studies on this feature for two qubits can be recovered from our studies as a special case. We also study the nonlocality of quantum states under collective dephasing. We find that although genuine entanglement of quantum states may not change, however their nonlocality changes. We discuss the possibility of finite time end of genuine nonlocality.
\end{abstract}

Quantum entanglement and nonlocality are features of quantum mechanics not only related to its foundation but also have applications in current and future technologies [1]3. Due to growing efforts for an experimental realization of devices utilizing these features, it is essential to 'study the effects of noisy environments on quantum correlations. Such studies are an active area of research [4] ' and several authors have studied decoherence effects on quantum correlations for both bipartite and multipartite systems [5] [16].

One specific type of noise dominant in experiments on trapped atoms is caused by intensity fluctuations of electromagnetic fields which leads to collective dephasing process. The detrimental effects of collective dephasing noise on entanglement have been studied [17 22], however all these previous studies were restricted to a special orientation (z-axes) of the field. Recently, a more general approach has been worked out [23, 24, where the authors addressed an arbitrary orientation of field. This general approach revealed an interesting feature of its dynamical process which is so called freezing dynamics of entanglement. It was shown that a specific two qubits state under certain orientation of the field may first decay upto some numerical value before suddenly stop decaying and main- tain this stationary entanglement 23. Such behavior was also predicted for multipartite states. Recently, we have confirmed this freezing entanglement phenomenon for various genuinely entangled states of three and four qubits, including random states [25]. Another interesting dynamical feature under this type of decoherence is the possibility of completely time-invariant entanglement, however time-invariance phenomenon so far including this work has only been observed for a special orientation of field (zaxes). Time-invariant entanglement does not necessarily mean that the quantum states live in decoherence free subspaces (DFS). In fact the quantum states may change at every instance whereas their entanglement remain constant throughout the dynamical process. This feature was first observed for qubit-qutrit systems [20] and more recently for qubit-qubit systems [22]. In this Letter, we investigate the time-invariant phenomenon for genuine entanglement of multiqubit quantum systems. It is known that genuine entanglement is different than the entanglement among bipartition and this type of entanglement is only a peculiar feature of multipartite quantum states. We have looked for this phenomenon in Hilbert space of three qubits and our preliminary search suggests that it may not exist for this dimension of Hilbert space. However, we 
have explicitly observed this phenomenon for a family of quantum states of four qubits. The interesting difference between three and four qubit case is the fact that for three qubits, all off-diagonal elements of GHZ-diagonal states decay and there are no DFS for them, whereas for four qubits, there are some GHZ-diagonal states which live in DFS. We have detected this phenomenon by taking mixtures of GHZ states living in DFS and ones living in other orthogonal subspaces. The mixing probability for entangled state preserved in DFS must be larger than the probability of entangled state which decay, such that although combined states might change whereas their entanglement stay invariant. It is interesting that genuine entanglement also exibits time-invariance even though the entanglement among bipartition is not constant as evident by a change in the negative eigenvalues of the partially transposed matrix. Recent progress in the theory of multipartite entanglement has enabled us to study decoherence effects on actual multipartite genuine entanglement and not on entanglement among bipartitions. In particular, the ability to compute genuine negativity for multipartite systems has eased this task 26].

Another concept related to non-classical correlations is quantum nonlocality. This feature says that the predictions made using quantum mechanics cannot be simulated by a local hidden variable model. The presence of nonlocal correlations can be detected via violation of some type of Bell inequalities 27. The pure entangled states violate a Bell inequality, whereas mixed entangled states may not do so 28 . However, all entangled states do exhibit some kind of hidden nonlocality 29. The well known Clauser-Horne-Shimony-Holt (CHSH) inequality 30 for two qubits has been studied under decoherence both in theory [31, and experiment 32. Several investigations of nonlocality of multipartite quantum states under decoherence have been carried out 33. The extension of $\mathrm{CHSH}$ inequality for multipartite quantum systems has received considerable attention 34 37, however Svetlichny discovered the first method to detect genuine multipartite nonlocality 38. Violations of some of these inequalities in experiments have been reported [39, 40]. We have studied the effect of collective dephasing on genuine nonlocality of quantum states exhibiting time-invariant dynamics. We have found that these quantum states may loose their genuine nonlocality at a finite time.

We review the basic notions of genuine multipartite entanglement and genuine nonlocality only for three parties $A, B$, and $C$. One can generalize these methods to more parties straightforwardly. A state is called separable with respect to some bipartition, say, $A \mid B C$, if it is a mixture of product states with respect to this partition, that is, $\rho=\sum_{j} p_{j}\left|\psi_{A}^{j}\right\rangle\left\langle\psi_{A}^{j}|\otimes| \psi_{B C}^{j}\right\rangle\left\langle\psi_{B C}^{j}\right|$, where $p_{j}$ form a probability distribution. We denote these states as $\rho_{A \mid B C}^{s e p}$. Similarly, we can define separable states for the two other bipartitions, $\rho_{B \mid C A}^{s e p}$ and $\rho_{C \mid A B}^{s e p}$. Then a state is called biseparable if it can be written as a mixture of states which are separable with respect to different bipartitions, that is

$$
\rho^{b s}=\tilde{p}_{1} \rho_{A \mid B C}^{s e p}+\tilde{p}_{2} \rho_{B \mid C A}^{s e p}+\tilde{p}_{3} \rho_{C \mid A B}^{s e p},
$$

with $\tilde{p}_{1}+\tilde{p}_{2}+\tilde{p}_{3}=1$. Finally, a state is called genuinely multipartite entangled if it is not biseparable. In the rest of this paper, we always mean genuine multipartite entanglement when we talk about entanglement.

Genuine entanglement can be detected and characterized 26 by a technique based on positive partial transpose mixtures (PPT mixtures). A two-party state $\rho=$ $\sum_{i j k l} \rho_{i j, k l}|i\rangle\langle j|\otimes| k\rangle\langle l|$ is PPT if its partially transposed matrix $\rho^{T_{A}}=\sum_{i j k l} \rho_{j i, k l}|i\rangle\langle j|\otimes| k\rangle\langle l|$ is positive semidefinite. The separable states are always PPT 41 and the set of separable states with respect to some partition is therefore contained in a larger set of states which has a positive partial transpose for that bipartition.

Denoting PPT states with respect to fixed bipartition by $\rho_{A \mid B C}^{P P T}, \rho_{B \mid C A}^{P P T}$, and $\rho_{C \mid A B}^{P P T}$, we call a state as PPT-mixture if it can be written as

$$
\rho^{P P T m i x}=q_{1} \rho_{A \mid B C}^{P P T}+q_{2} \rho_{B \mid C A}^{P P T}+q_{3} \rho_{C \mid A B}^{P P T} .
$$

As any biseparable state is a PPT-mixture, therefore any state which is not a PPT-mixture is guaranteed to be genuinely multipartite entangled. The main advantage of considering PPT-mixtures instead of biseparable states comes from the fact that PPT-mixtures can be fully characterized by the method of semidefinite programming (SDP), a standard method in convex optimization [42. Generally the set of PPT-mixtures is a very good approximation to the set of biseparable states and delivers the best known separability criteria for many cases; however, there are multipartite entangled states which are PPT-mixtures 26. In order to quantify genuine multipartite entanglement, it was shown [26] that for the following optimization problem

$$
\min \operatorname{Tr}(\mathcal{W} \rho)
$$

with constraints that for all bipartition $M \mid \bar{M}$

$\mathcal{W}=P_{M}+Q_{M}^{T_{M}}$, with $0 \leq P_{M} \leq \mathbb{I}$ and $0 \leq Q_{M} \leq \mathbb{I}$

the negative witness expectation value is multipartite entanglement monotone. The constraints just state that the considered operator $\mathcal{W}$ is a decomposable entanglement witness for any bipartition. Since this is a semidefinite program, the minimum can be efficiently computed and the optimality of the solution can be certified 42. We denote this measure by $E(\rho)$ or $E$-monotone in this paper. For bipartite systems, this monotone is equivalent to negativity 43 . For a system of qubits, this measure is bounded by $E(\rho) \leq 1 / 2$ [4].

For a brief description of genuine nonlocality, consider that each party can perform a measurement $X_{j}$ with result $a_{j}$ for $j=A, B, C$. The joint probability distribution $P\left(a_{A} a_{B} a_{C} \mid X_{A} X_{B} X_{C}\right)$ may exhibit different notions 
of nonlocality. It may be that it cannot be written in local form as

$$
\begin{array}{r}
P\left(a_{A} a_{B} a_{C} \mid X_{A} X_{B} X_{C}\right)=\int d \lambda p_{\lambda} P_{A}\left(a_{A} \mid X_{A} \lambda\right) \\
P_{B}\left(a_{B} \mid X_{B} \lambda\right) P_{C}\left(a_{C} \mid X_{C} \lambda\right),
\end{array}
$$

where $\lambda$ is a shared local variable. Such nonlocality can be tested by standard Bell inequalities and it can not capture the genuine nonlocality. As an example consider that parties $A$ and $B$ are nonlocally correlated but uncorrelated from party $C$. It is still possible that $P$ cannot be written as Eq.(5), although the system has no genuine tripartite nonlocality [37. Genuine nonlocality can be detected if one makes sure that $P$ cannot be written as

$$
\begin{array}{r}
P_{G}\left(a_{A} a_{B} a_{C} \mid X_{A} X_{B} X_{C}\right)=\sum_{m=1}^{3} p_{m} \int d \lambda \rho_{i j}(\lambda) \\
P_{i j}\left(a_{i} a_{j} \mid X_{i} X_{j} \lambda\right) P_{m}\left(a_{m} \mid X_{m} \lambda\right),
\end{array}
$$

that is, $P$ cannot be reproduced by local means even if any two of parties come together and act jointly to produce bipartite nonlocal correlations with probability distribution $\rho_{i j}(\lambda)$, where $i j$ denotes for all possible partitions. We focuss on the possibility that each party $j$ is allowed to two measurements $X_{j}$ and $X_{j}^{\prime}$ with outcomes $a_{j}$ and $a_{j}^{\prime}$ such that $a_{j}, a_{j}^{\prime} \in\{-1,1\}$. For an initial state $|G H Z\rangle=(|0000\rangle+|1111\rangle) / \sqrt{2}$, we consider the Ardehali inequality [35] $\left\langle B_{A}\right\rangle \leq 4$, where

$$
\begin{aligned}
\mathcal{B}_{A}= & \left(A_{1} X_{2} X_{3} X_{4}+B_{1} X_{2} X_{3} X_{4}-\left[A_{1} X_{2} Y_{3} Y_{4}+\text { perm }\right]\right. \\
& -\left[B_{1} X_{2} Y_{3} Y_{4}+\text { perm }\right]-\left[A_{1} X_{2} X_{3} Y_{4}+\text { perm }\right] \\
& \left.+\left[B_{1} X_{2} X_{3} Y_{4}+\text { perm }\right]+A_{1} Y_{2} Y_{3} Y_{4}-B_{1} Y_{2} Y_{3} Y_{4}\right),
\end{aligned}
$$

and the sum in square brackets include all distinct permutations on last three qubits, $A_{1}=\left(X_{1}+Y_{1}\right) / \sqrt{2}$, and $B_{1}=\left(X_{1}-Y_{1}\right) / \sqrt{2}$. The expectation value for GHZ state is $\left\langle B_{A}\right\rangle=8 \sqrt{2}$, which is the maximum violation for four qubits.

We consider our qubits as atomic two-level systems with energy splitting $\hbar \omega$. The splitting is controlled by a homogeneous magetic field. The Hamiltonian for a single atom is given as

$$
\hat{H}_{\omega}=\frac{\hbar \omega}{2} \vec{n} \cdot \vec{\sigma}
$$

where $\vec{n}=n_{x} \hat{x}+n_{y} \hat{y}+n_{z} \hat{z}$ is the orientation of magnetic field and $\vec{\sigma}=\sigma_{x} \hat{x}+\sigma_{y} \hat{y}+\sigma_{z} \hat{z}$ is the vector of Pauli matrices. This time independent Hamiltonian generates the propagator

$$
U_{\omega}(t)=\mathrm{e}^{-i H_{\omega} t / \hbar}=\mathrm{e}^{-i \omega t / 2 \mathbf{n} \cdot \sigma} .
$$

We can introduce a pair of orthogonal projectors

$$
\Lambda_{ \pm}=\frac{I_{2} \pm \mathbf{n} \cdot \sigma}{2}
$$

to write the propagator in terms of them. Let us consider $N$ non-interacting atoms (qubits), so that the propagator for these collection of atoms can be written as 23.

$$
\begin{aligned}
U_{\omega}(t)^{\otimes N}= & \left(e^{-i \omega t} \Lambda_{+}+e^{i \omega t} \Lambda_{-}\right)^{\otimes N} \\
& =\sum_{j=0}^{N} e^{i \omega t(j-N / 2)} \Theta_{j},
\end{aligned}
$$

where the operators $\Theta_{j}$ are defined as

$$
\Theta_{j}=\frac{1}{j !(N-j) !} \sum_{s \in \sum_{N}} V_{s}\left[\Lambda_{-}^{\otimes j} \otimes \Lambda_{+}^{\otimes N-j}\right] V_{s}^{\dagger},
$$

where $\sum_{N}$ represents the symmetric group and $V_{s}$ are the permutations in operator space of $N$ qubits.

As there are fluctuations in the magetic field strength, the integration over it will induce a probability distribution $p(\omega)$ of characteristic energy splitting. Therefore the time evolution of the combined state of $N$ atoms can be written as 23

$$
\rho(t)=\int p(\omega) U_{\omega}(t)^{\otimes N} \rho(0) U_{\omega}^{\dagger}(t)^{\otimes N} d \omega .
$$

In writing this equation, we have assumed that the field fluctuations occur on time scale which are longer than the time over which the combined state of $N$ atoms evolve under unitary propagator $U_{\omega}(t)^{\otimes N}$. Substituting the above derived format for the unitary propagator, we can write the time evolved state as

$$
\rho(t)=\sum_{j, k=0}^{N} M_{j k}(t) \Theta_{j} \rho(0) \Theta_{k},
$$

where $M_{j k}(t)$ are elements of the Toeplitz matrix $M(t)$, which can be obtained by the relation $M_{j k}(t)=\phi[(j-k) t]$, where $\phi(t)$ is the characteristic function of the probability distribution $p(\omega)$, defined as

$$
\phi(t)=\int p(\omega) e^{i \omega t} d \omega .
$$

It has been demonstrated that time evolution form Eq.(14) is both trace preserving and positivity preserving 23. In order to study the exact behavior of multipartite quantum states, it is convenient to obtain an exact expression for state $\rho(t)$, in terms of a spectral distribution $p(\omega)$ characterizing the fluctuations. As an example, we take the Lorentzian distribution also known as Cauchy distribution, defined as

$$
p(x)=\frac{\gamma^{2}}{\pi \gamma\left[\left(x-x_{0}\right)^{2}+\gamma^{2}\right]} .
$$

For standard Cauchy distribution, the characteristic function turns out to be

$$
\phi(t)=\int p(x) e^{i x t} d x=e^{-|t|},
$$


here $t$ denotes dimensionless quantity usually taken as $\Gamma t$. The time evolution of an arbitrary initial state can be obtained straightforwardly. In general there are no decoherence free subspaces (DFS) in this noisy model except for some special directions of field, like $\vec{n}=(0,0,1)^{T}$, etc. In addition it may happen that some quantum states are completely invariant for certain directions of field as well. However, an interesting and non-trivial possibility is the time-invariant entanglement such that the quantum states are changing at every instance however their entanglement remains constant. Such observation was initially made for qubit-qutrit systems [20] and later on for a specific family which is so called Bell-diagonal states of two qubits [22]. The time-invariant entanglement phenomenon including our current study has been observed for $\vec{n}=(0,0,1)^{T}$. This direction of field always have DFS for all dimensions of Hilbert space. The combination of quantum states residing in DFS and subspaces orthogonal to them lead to this phenomenon as explained below.

By choosing $\vec{n}=(0,0,1)^{T}$ onwards in this Letter, first we take an example of two qubits. The resulting time evolved quantum state for two qubits can be obtained straightforwardly as

$$
\rho(t)=\left(\begin{array}{cccc}
\rho_{11} & \gamma \rho_{12} & \gamma \rho_{13} & \gamma^{2} \rho_{14} \\
\gamma \rho_{21} & \rho_{22} & \rho_{23} & \gamma \rho_{24} \\
\gamma \rho_{31} & \rho_{32} & \rho_{33} & \gamma \rho_{34} \\
\gamma^{2} \rho_{41} & \gamma \rho_{42} & \gamma \rho_{43} & \rho_{44}
\end{array}\right),
$$

where $\gamma=e^{-\Gamma t}$. We note that there are two sectors in which entangled states can reside. For Bell states $\left|\Phi^{ \pm}\right\rangle=1 / \sqrt{2}(|00\rangle \pm|11\rangle)$, there is decay of entanglement, whereas Bell states $\left|\Psi^{ \pm}\right\rangle=1 / \sqrt{2}(|01\rangle \pm|10\rangle)$ remain invariant while being in DFS. If we mix any Bell state living in DFS with any other Bell state in orthogonal subspace then we may find the phenomenon of time-invariant entanglement. As a concrete example, let us consider the family of states

$$
\rho_{a, b}=b\left|\Psi^{ \pm}\right\rangle\left\langle\Psi^{ \pm}\right|+(1-b) \rho_{a},
$$

where $0 \leq b \leq 1$ and $\rho_{a}$ is defined as

$$
\rho_{a}=a\left|\Phi^{+}\right\rangle\left\langle\Phi^{+}\right|+\frac{1-a}{4} \mathbb{I}_{4},
$$

where $0 \leq a \leq 1$. The time evolution of states $\rho_{a, b}$ can be straightforwardly written as

$$
\rho_{a, b}(t)=b\left|\Psi^{ \pm}\right\rangle\left\langle\Psi^{ \pm}\right|+(1-b) \rho_{a}(t) .
$$

The four eigenvalues of the partially transposed matrix $\rho_{a, b}^{T_{A}}(t)$ are $[1+a-(3+a) b] / 4,[1+a+b-a b] / 4,[1+b-a(1-$ b) $\left.\left(1-2 e^{-2 \Gamma t}\right)\right] / 4$, and $\left[1+b-a(1-b)\left(1+2 e^{-2 \Gamma t}\right)\right] / 4$. As it is known that for two qubits, the partially transposed matrix can have maximum one negative eigenvalue, therefore for the choice

$$
b>\frac{1+a}{3+a},
$$

the first eigenvalue is negative and the rest of the three eigenvalues are positive. As this negative eigenvalue is time-invariant, therefore we can quantify entanglement by negativity as

$$
N\left(\rho_{a, b}(t)\right)=\frac{(3+a) b-1-a}{2},
$$

causing time-invariant entanglement although the quantum states are changing at every instance as evident by their eigenvalues. If condition (22) is not satisfied then entanglement decays. As a special case of $a=1, b=0.7$ and $b=0.75$, we recover the results already worked out recently 22 .

Let us consider three qubits. In this case, we have two types of inequivalent genuinely entangled states, namely $G H Z$ type states and $W$ type state. The most general solution for an arbitrary initial state is similar to Eq. (18), such that $W$ state and $\tilde{W}$ (locally equivalent state) reside in decoherence free subspace. The natural extension of Bell-diagonal states for multi qubits are GHZ-diagonal states. In computational basis these states lie on the main diagonal and anti-diagonal of density matrix, hence forming an X just like Bell-diagonal states. The GHZ-diagonal states are subset of $\mathrm{X}$-states and all off-diagonal matrix elements decay under collective dephasing. Therefore in order to look for time-invariant entanglement we must take mixture of GHZ states and W states. The only GHZ state $|G H Z\rangle=(|000\rangle \pm|111\rangle) / \sqrt{2}$ has no overlap with W state, so we define our quantum states as

$$
\rho_{\eta}=(1-\eta)|G H Z\rangle\langle G H Z|+\eta| W\rangle\langle W|,
$$

where $0 \leq \eta \leq 1$ and $|W\rangle=1 / \sqrt{3}(|001\rangle+|010\rangle+|100\rangle)$. The time evolved states are written as

$$
\rho_{\eta}(t)=(1-\eta)|G H Z(t)\rangle\langle G H Z(t)|+\eta| W\rangle\langle W| .
$$

As it is hard to find analytical expressions for genuine negativity, nevertheless, based on our numerical search, we conjecture that there is no time-invariant genuine entanglement for three qubits under collective dephasing. Figure (10) shows the genuine negativity for two values of parameter $\eta$. We see that although entanglement is changing at a very slow rate due to very large percentage of $W$ state, nevertheless, we do not have any time-invariant entanglement. Our numerical calculations suggests that we do not have any time-invariant entanglement even for $\eta=0.99$.

Finally we move to four qubits case. We demonstrate explicitly that time-invariant entanglement can occur for this dimension of Hilbert space. The sixteen GHZ states in this case are defined as

$$
\left|G H Z_{i}\right\rangle=\frac{\left|x_{1} x_{2} x_{3} x_{4}\right\rangle \pm\left|\bar{x}_{1} \bar{x}_{2} \bar{x}_{3} \bar{x}_{4}\right\rangle}{\sqrt{2}},
$$

where $x_{j}, \bar{x}_{j} \in\{0,1\}$ and $x_{j} \neq \bar{x}_{j}$. We note that in contrast to the three qubits, four qubits case do have some 


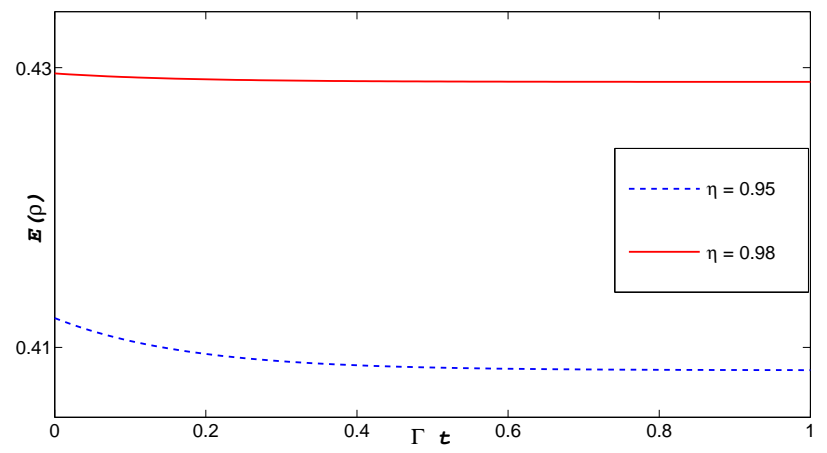

Fig. 1: Genuine negativity for three qubits is plotted against parameter $\Gamma t$ for $\rho_{\eta}(t)$ states (Eq. (25) ) for two values of parameter $\eta$. See text for details.

GHZ states living in DFS. More specifically, all those GHZ states which have two 1's in a ket reside in DFS, for an example, the state $(|0011\rangle \pm|1100\rangle) / \sqrt{2}$, and other states with permutations. We are now in a position to define a family of quantum states similar to two qubits case as

$$
\rho_{\alpha}=\alpha\left|G H Z_{2}\right\rangle\left\langle G H Z_{2}\right|+\frac{1-\alpha}{16} \mathbb{I}_{16},
$$

where $\left|G H Z_{2}\right\rangle=(|0001\rangle+|1110\rangle) / \sqrt{2}$ and $0 \leq \alpha \leq 1$. We take a mixture of this state with a state which resides in DFS, given as

$$
\rho_{\alpha, \beta}=\beta\left|G H Z_{6}\right\rangle\left\langle G H Z_{6}\right|+(1-\beta) \rho_{\alpha},
$$

where $\left|G H Z_{6}\right\rangle=(|0101\rangle+|1010\rangle) / \sqrt{2}$ and $0 \leq \beta \leq 1$. The time evolution of these states can be written as

$$
\rho_{\alpha, \beta}(t)=\beta\left|G H Z_{6}\right\rangle\left\langle G H Z_{6}\right|+(1-\beta) \rho_{\alpha}(t) .
$$

We can analyze the eigenvalues of the partially transposed matrix $\rho_{\alpha, \beta}^{T_{A}}(t)$, however we should remember the fact that there are states which are NPT under each partition nevertheless they are biseparable [45. Although Figure (33) can be regarded as manisfestation of explicit time dependence of quantum states, nevertheless, the spectrum of quantum states $\rho_{\alpha, \beta}(t)$ can also show their explicit time dependence. The eigenvalues of these states are $(1-\alpha)(1-\beta) / 16(13$ times), $(1-\alpha+15 \beta+\alpha \beta) / 16,\left(1+7 \alpha-8 \alpha e^{-2 \Gamma t}\right)(1-$ $\beta) / 16$, and $\left(1+7 \alpha+8 \alpha e^{-2 \Gamma t}\right)(1-\beta) / 16$. The last two eigenvalues are time dependent and exhibit the fact that quantum states are changing at all times.

Figure 2 shows genuine negativity plotted against parameter $\Gamma t$ for family of states $\rho_{\alpha, \beta}(t)$. We have set $\alpha=0.9$ and plotted three instances for parameter $\beta$. We can see that for $\beta=0.85$ and $\beta=0.8$, we have time-invariant genuine entanglement, whereas for $\beta=0.1$, we have decay of entanglement. For smaller values of $\beta$, the subspace orthogonal to DFS is dominant and entanglement decays, whereas for larger values of $\beta$, we may get time-invariant entanglement even though the quantum

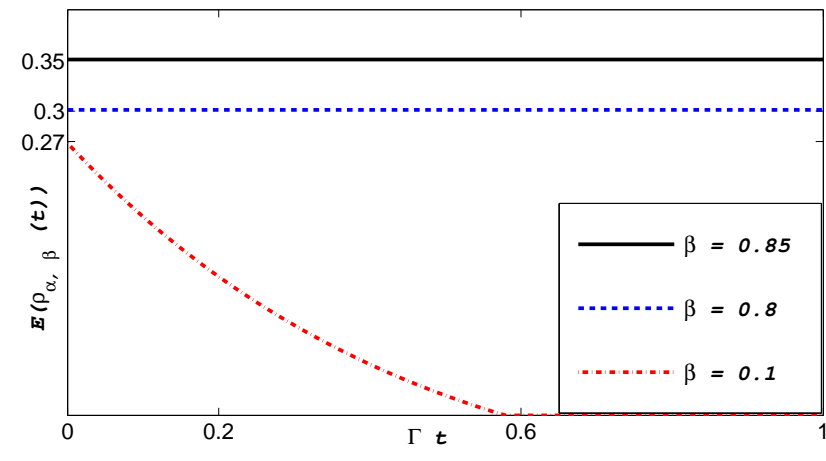

Fig. 2: Genuine negativity for four qubits is plotted against parameter $\Gamma t$ for $\rho_{\alpha, \beta}(t)$ states (Eq. (29)) for various values of parameter $\beta$. We take $\alpha=0.9$. See text for details.

states are changing at every instance. Hence we have explicitly demonstrated the existance of time-invariant feature for genuine entanglement of four qubits.

In order to study the genuine nonlocality of states $\rho_{\alpha, \beta}(t)$, we need to find appropriate measurement operators for it. As time-invariant entanglement occurs for larger values of $\beta$, which implies that the largest offdiagonal matrix element corresponds to state $\left|G H Z_{6}\right\rangle$. As all GHZ states are locally equivalent to each other via a local unitary operator, therefore we can apply same local unitary transformations to measurement operators and then take expectation value of Bell operator $\mathcal{B}_{A}$. The expectation value of such operator is given as

$$
\left\langle\mathcal{B}_{A}\right\rangle=\frac{16 \beta-\alpha(1-\beta)\left(9-7 e^{-2 \Gamma t}\right)}{\sqrt{2}} .
$$

We note that for $\beta=1$, we have maximum violation of $8 \sqrt{2}$, which is expected as the state is in DFS and not changing so its genuine nonlocality remains invariant. Whereas for $\beta<1$, we have decay of genuine nonlocality provided that $\alpha \neq 0$. Depending upon parameter $\beta$, we can either have nonlocal states throughout the dynamics or sudden death of genuine nonlocality. We find that for

$$
\beta>\frac{4 \sqrt{2}+\alpha}{8+\alpha},
$$

we have initial genuine nonlocal quantum states. It is well known that the $n$-partite quantum state $\rho(t)$ exibits genuine multipartite nonlocality if $|\langle\mathcal{B}\rangle|>2^{n-1}$. In Figure (3), we plot the expectation value of $\mathcal{B}_{A}$ against parameter $\Gamma t$ for $\beta=0.85$ (black solid line) and $\beta=0.8$ (blue dashed line). We see that we have asymptotic nonlocal states for top curve and sudden death of nonlocality for lower curve. In both situations, these curves indicate the fact that quatum states are changing at every instance, such that genuine nonlocality is changing whereas its genuine entanglement does not change at all.

In summary we have studied the dynamics of genuine entanglement and genuine nonlocality for multiqubits 


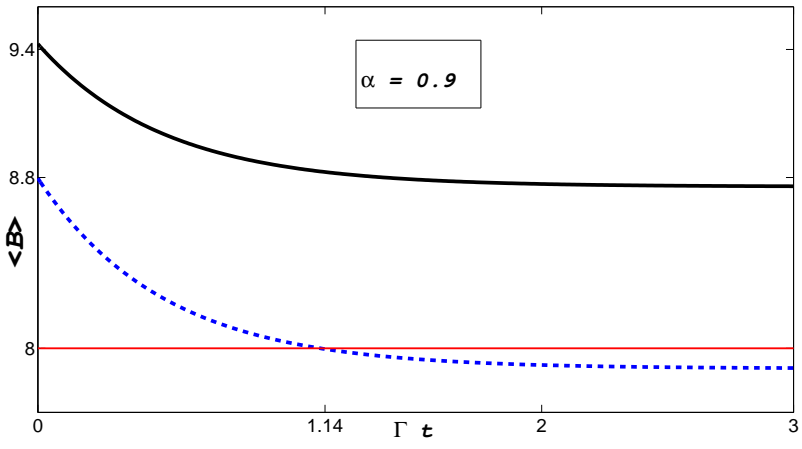

Fig. 3: Genuine nonlocality is plotted against parameter $\Gamma t$ for states $\rho_{\alpha, \beta}(t)$. We take $\alpha=0.9$. Top curve is for $\beta=0.85$ and depicts asymptotic nonlocal states. The lower curve is for $\beta=0.8$ and shows sudden death of genuine nonlocality.

quantum systems under collective dephasing. We have investigated the possibility of time-invariant entanglement for two, three and four qubits. We have found that there exist a non-trivial feature of quantum states, in which the states are changing at every instance whereas their entanglement remain constant. The change in quantum states might be indicated by the change in their nonlocality. This feature was first discovered for qubit-qutrit systems and later on for qubit-qubit quantum states. Based on our numerical search, we conjecture that there is no evidence of time-invariant entanglement for three qubits states. For four qubits, we have explicitly demonstrated phenomenon of time-invariant genuine entanglement for a family of quantum states. It seems that if we have mixtures of two entangled states such that one entangled state lives in decoherence free subspace and other entangled state resides in orthogonal subspaces to it, only then we might observe this phenomenon. The fraction of entangled states living in DFS must be larger than entangled states in orthogonal subspaces. We have also studied the decoherence effects on genuine nonlocality of those quantum states which exhibit time-invariant entanglement. We have found that these states may exhibit either asymptotic nonlocality or so called sudden death of nonlocality, in which quantum states loose their nonlocality at a finite time. Although the quantum states remain genuine entangled, nevertheless, their genuine nonlocality varies. We believe that our findings can be verified in already available ion trap or photonic experiments, for example, in ${ }^{40} \mathrm{Ca}^{+}$ ion, the operation of $M S_{\phi}(\pi / 2)$ can map the ground state $|0000\rangle$ of four qubits directly to maximally entangled GHZ state [46. Any other GHZ state can be obtained by rotation $R_{\phi}(\pi / 2)$. The state $\rho_{\alpha}$ (Eq. (27) ) can be prepared via same technique as Werner states have been prepared with operation $M S 2=\exp \left(-i \pi / 4 \sigma_{J} \sigma_{J}\right)$ using $854 \mathrm{~nm}$ and $729 \mathrm{~nm}$ pulses 47] and so on. Experimental verification of time-invariant entanglement of two qubits [22] indicates the interest and importance of such experiments for mul- tipar te quantum systems. Finally, one may ask how the resul obtained here may change if we have local decoherence nstead of global. As we have pointed out that the prese ice of decoherence free subspaces seems to be a necessary ondition for the time-invariant entanglement and it is $\mathrm{kn}$ wn that for local dephasing there are no decoherence free ibspaces, therefore, we expect no such phenomenon in th $t$ case. Our previous studies on dynamics of genuine ental slement for local decoherence also support this claim [16. One of the future avenues would be to look for the time- nvariance of quantum nonlocality.

$$
* * *
$$

M. Ali is grateful to Edoardo G. Carnio for helpful discussions and Otfried Gühne for his correspondence. The author is also thankful to both referees for their constructive and helpful comments which brought much clarity in the Letter.

\section{REFERENCES}

[1] Horodecki R et al., Rev. Mod. Phys., 81 (2009) 865.

[2] Gühne O and Tóth G, Phys. Rep., 474 (2009) 1.

[3] Brunner N., Cavalcanti D., Pironio S., Scarani V and Wehner S., Rev. Mod. Phys., 86 (2014) 419.

[4] Aolita L., de Melo F. and Davidovich L., Rep. Prog. Phys., 78 (2015) 042001.

[5] Yu T. and Eberly J. H., Phys. Rev. Lett., 93 (2004) 140404.

[6] Dür W. and Briegel H. J., Phys. Rev. Lett., 92 (2002) 180403; Hein M., Dür W. and Briegel H. J., Phys. Rev. A, 71 (2005) 032350.

[7] Aolita L. et al., Phys. Rev. Lett., 100 (2008) 080501.

[8] Simon C. and Kempe J., Phys. Rev. A, 65 (2002) 052327; Borras A. et al., Phys. Rev. A, 79 (2009) 022108; CavAlcanti D. et al., Phys. Rev. Lett., 103 (2009) 030502.

[9] Bandyopadhyay S. and Lidar D. A., Phys. Rev. A, 72 (2005) 042339; Chaves R. and Davidovich L., Phys. Rev. A, 82 (2010) 052308; Aolita L. et al., Phys. Rev. A, 82 (2010) 032317.

[10] Carvalho A. R. R., Mintert F. and Buchleitner A., Phys. Rev. Lett., 93 (2004) 230501.

[11] Lastra F., Romero G., Lopez C. E., França Santos M. and Retamal J. C., Phys. Rev A, 75 (2007) 062324.

[12] Gühne O, Bodoky F and Blaauboer M., Phys. Rev. A, 78 (2008) 060301(R).

[13] López C. E., Romero G., Lastra F., Solano E. and Retamal J. C., Phys. Rev. Lett., 101 (2008) 080503.

[14] Rau A. R. P., Ali M. and Alber G., EPL, 82 (2008) 40002; Ali M., Alber G. and Rau A. R. P., J. Phys. B: At. Mol. Opt. Phys., 42 (2009) 025501; Ali M., J. Phys. B: At. Mol. Opt. Phys., 43 (2010) 045504.

[15] Weinstein Y. S. et al., Phys. Rev. A, 85 (2012) 032324; Filippov S. N., Melnikov A. A. and Ziman M., Phys. Rev. A, 88 (2013) 062328.

[16] Ali M. and Gühne O., J. Phys. B: At. Mol. Opt. Phys., 47 (2014) 055503; Ali M., Phys. Lett. A, 378 (2014) 2048; Ali M. and Rau A. R. P., Phys. Rev. A, 90 (2014) 
042330; Ali M., Open. Sys. \& Info. Dyn., 21 (2014) 1450008.

[17] Yu T. and Eberly J. H., Phys. Rev. B, 66 (2002) 193306; Yu T. and Eberly J. H., Phys. Rev. B, 68 (2003) 165322; Yu T. and Eberly J. H., Optics Communications, 264 (2006) 393.

[18] Jaeger G. and Ann K., J. Mod. Opt., 54 (2007) 2327.

[19] Li S-B. and Xu J-B., Eur. Phys. J. D., 41 (2007) 377.

[20] Karpat G. and Gedik Z., Phys. Lett. A, 375 (2011) 4166.

[21] Ali M., Phys. Rev. A, 81 (2010) 042303; Ali M., Chin. Phys. Lett., 32 (2015) 060302.

[22] Liu B-H. et al., Phys. Rev. A, 94 (2016) 062107.

[23] Carnio E. G., Buchleitner A. and Gessner M., Phys. Rev. Lett., 115 (2015) 010404.

[24] Carnio E. G., Buchleitner A. and Gessner M., New. J. Phys., 18 (2016) 073010.

[25] Ali M., Preprint, (2016).

[26] Jungnitsch B., Moroder T. and Gühne O., Phys. Rev. Lett., 106 (2011) 190502; Novo L., Moroder T. and Gühne O., Phys. Rev. A, 88 (2013) 012305; Hofmann M., Moroder T. and Gühne O., J. Phys. A: Math. Theor., 47 (2014) 155301.

[27] Bell J. S., Physics, 1 (1964) 195.

[28] Gisin N., Phys. Lett. A, 154 (1991) 201; Werner R. F., Phys. Rev. A, 40 (1989) 4277.

[29] Liang Y-C., Masanes L. and Rosset D., Phys. Rev. A, 86 (2011) 052115.

[30] Clauser J. F., Horne M. A., Shimony A. and Holt R. A., Phys. Rev. Lett., 23 (1969) 880.

[31] Mazzola L., Bellomo B., Lo Franco R. and ComPagno G., Phys. Rev. A, 81 (2010) 052116.

[32] Xu J-S. et al., Phys. Rev. Lett., 104 (2010) 100502.

[33] Laskowski W., Paterek T., Brukner C. and Zukowski M., Phys. Rev. A, 81 (2010) 042101; Chaves R., Cavalcanti D., Aolita L. and Acin A., Phys. Rev. A, 86 (2012) 012108; Chaves R., Acin A., Aolita L. and Cavalcanti D., Phys. Rev. A, 89 (2014) 042106; Sohbi A., Zaquine I., Diamanti E. and Markham D., Phys. Rev. A, 91 (2015) 022101; Divianszky P., Trencsenyi R., Bene E. and Vertesi T., Phys. Rev. A, 93 (2016) 042113; Laskowski W., Vertesi T. and WiesNiAK M., J. Phys. A: Math. Theor., 48 (2015) 465301.

[34] Mermin D., Phys. Rev Lett., 65 (1990) 1838.

[35] Ardehali M., Phys. Rev. A, 46 (1992) 5375.

[36] Collins D., Gisin N., Popescu S., Roberts D. and Scarani V., Phys. Rev Lett., 88 (2002) 170405.

[37] Bancal J-D., Brunner N., Gisin N. and Liang Y-C., Phys. Rev. Lett., 106 (2011) 020405.

[38] Svetlichny G., Phys. Rev. D, 35 (1987) 3066.

[39] Pan J. W. et al., Nature, 403 (2000) 515; Lavoie J., Kaltenbaek R. and Resch K. J., New. J. Phys., 11 (2009) 073051; ERWEN C. et al., Nature Photonics, 8 (2014) 292.

[40] Jungnitsch B. et al., Phys. Rev. Lett., 104 (2010) 210401.

[41] Peres A., Phys. Rev. Lett., 77 (1996) 1413.

[42] Vandenberghe L. and Boyd S., SIAM Rev., 38 (1996) 49.

[43] Vidal G. and Werner R. F., Phys. Rev. A, 65 (2002) 032314.

[44] Jungnitsch B., Moroder T. and Gühne O, Phys. Rev.
A, 84 (2011) 032310.

[45] Sabín C. and García-Alcaine G., Eur. Phys. J. D, 48 (2008) 435.

[46] Schindler P. et al., New. J. Phys., 15 (2013) 123012.

[47] Lanyon B. P., Jurcevic P., Hempel C., Gessner M., Vedral V., Blatt R. and Roos C. F., Phys. Rev. Lett., 111 (2013) 100504. 